
Horticultura Brasileira 27: 055-058.

\title{
Teste de envelhecimento acelerado para avaliação do potencial fisiológico de sementes de urucum
}

\author{
Salvador B Torres'; Francisco Bezerra Neto ${ }^{2}$ \\ 'EMPARN/UFERSA, C. Postal 137, 59625-900 Mossoró-RN; ²UFERSA, Progr. Pós-graduação fitotecnia; sbtorres@ufersa.edu.br
}

\begin{abstract}
RESUMO
Urucum (Bixa orellana L.) é uma cultura de grande interesse comercial, sendo o principal produto a semente, da qual se extraem os corantes bixina e norbixina, de valor nos mercados nacional e internacional. O presente trabalho teve por objetivo estudar a metodologia do teste de envelhecimento acelerado para obtenção do potencial fisiológico de sementes de urucum, utilizando-se quatro lotes de sementes da cultivar Casca Vermelha. A avaliação inicial desses lotes consistiu na determinação do grau de umidade, germinação, primeira contagem da germinação e emergência de plântulas em casa de vegetação. $\mathrm{O}$ envelhecimento acelerado foi implementado a $41^{\circ} \mathrm{C}$ durante 48,72 e 96 horas, com e sem uso de solução saturada de $\mathrm{NaCl}$. O experimento foi conduzido em delineamento inteiramente casualizado. Dentre os procedimentos adotados no teste de envelhecimento acelerado, o período de exposição de 72 horas a $41^{\circ} \mathrm{C}$ com uso de solução saturada de $\mathrm{NaCl}$, revelou-se adequado para a avaliação do potencial fisiológico de sementes de urucum.
\end{abstract}

Palavras-chave: Bixa orellana, vigor, germinação.

\begin{abstract}
Accelerated aging test to evaluate the physiologic potential of annatto seeds

Annatto (Bixa orellana L.) is a crop of great commercial interest, from whose main product, the seed, is extracted the bixina and norbixina coloring, of great interest in the national and international market. The methodology of the accelerated aging test to achieve the physiological quality of annatto seeds was evaluated. The initial quality of the seeds was obtained through the tests of moisture content, germination, germination first count and seedling emergence in greenhouse. The accelerated aging test was conducted at $41^{\circ} \mathrm{C}$ during 48, 72 and 96 hours, using the traditional and $\mathrm{NaCl}$ saturated solution. The research was conducted in a completely randomized design. The saturated salt accelerated aging test was efficient for vigor evaluation of annatto seeds, and the period of 72 hours at $41^{\circ} \mathrm{C}$ was considered as the most adequate procedure to evaluate seed vigor levels.
\end{abstract}

Keywords: Bixa orellana, vigor, germination.

\section{(Recebido para publicação em 1 de fevereiro de 2008; aceito em 27 de fevereiro de 2009)}

\section{(Received in February 1, 2008; accepted in February 27, 2009)}

$\mathrm{O}$ urucum, da família Bixaceae, originário da América e cultivado na África e na Ásia é uma espécie perene de importância nacional e regional. No Brasil é comumente encontrada como planta ornamental, pela beleza e colorido de suas flores, além de ser também considerada planta medicinal (Lorenzi, 2002). Suas sementes são valiosas pela característica de produzir pigmentos, que são utilizados como corante natural, nas indústrias alimentícias, farmacêutica, cosmética e avícola (Harder, 2005).

Dado o fato de que a utilização de sementes de alta qualidade constitui a base para elevação da produtividade agrícola, o componente fisiológico da qualidade de sementes tem sido objeto de inúmeras pesquisas, em decorrência das sementes estarem sujeitas a uma série de mudanças degenerativas após a sua maturidade. No entanto, para muitas espécies, como o urucum, a pesquisa ainda não indica procedimentos apropriados para avaliação do vigor de sementes que possam ser utilizados pelas empresas produtoras nos programas internos de controle de qualidade e/ou para caracterizar as sementes destinadas à comercialização.

Dentre os testes de vigor disponíveis na atualidade, o de envelhecimento acelerado é um dos mais utilizados para estimar o potencial relativo de armazenamento dos lotes de sementes, apresentando resultados relacionados à emergência de plântulas em campo (TeKrony, 1995). Este teste se fundamenta no aumento da deterioração das sementes, quando expostas às condições adversas de alta temperatura e umidade relativa elevada. Sob essas condições, sementes de baixa qualidade deterioramse mais rapidamente do que sementes mais vigorosas, estabelecendo diferenças no potencial fisiológico dos lotes avaliados (AOSA, 1983).

Dentre os fatores que afetam o comportamento das sementes submetidas ao teste, a interação temperatura/período de exposição é um dos mais importantes. Alguns autores têm-se dedicado ao es- tudo dessa interação, indicando, para sementes de cebola $41^{\circ} \mathrm{C} / 72 \mathrm{~h}$ (TeKrony, 1995); tomate, $41^{\circ} \mathrm{C} / 72 \mathrm{~h}$ (Panobianco \& Marcos Filho, 2001); melão, $38^{\circ} \mathrm{C}$ ou $41^{\circ} \mathrm{C} / 72 \mathrm{~h}$ e $96 \mathrm{~h}$ (Torres \& Marcos Filho, 2003); ervilha, $41^{\circ} \mathrm{C} / 48$ e $72 \mathrm{~h}$ (Nascimento et al., 2007); brássicas, $42^{\circ} \mathrm{C} / 48$ a $96 \mathrm{~h}$ (Costa et al., 2008) e urucu, $42^{\circ} \mathrm{C} / 96 \mathrm{~h}$ (Lopes et al., 2008). Portanto, verificase que há tendência entre as pesquisas para a utilização das temperaturas de 41 e $42^{\circ} \mathrm{C}$.

Outro aspecto a ser considerado no teste de envelhecimento acelerado, são as diferenças na absorção de água pelas sementes que, quando expostas à atmosfera úmida, podem apresentar variações acentuadas no grau de umidade. Pesquisas conduzidas com espécies de sementes pequenas têm revelado resultados pouco consistentes devido à variação muito acentuada do grau de umidade das amostras, após o envelhecimento (Powell, 1995). Para contornar esse problema, foi sugerida por Jianhua \& McDonald (1996) a substituição da 
Tabela 1. Germinação, primeira contagem e emergência de plântulas em casa de vegetação de sementes de urucum, cultivar Casca Vermelha (germination, first counting and seedling emergence in the greenhouse percentage of annatto seeds, cultivar Casca Vermelha). Mossoró, UFERSA, 2007.

\begin{tabular}{lccc}
\hline Lote & $\begin{array}{c}\text { Germinação } \\
(\%)\end{array}$ & $\begin{array}{c}\text { Primeira } \\
\text { contagem (\%) }\end{array}$ & $\begin{array}{c}\text { Emergência de plântulas em casa } \\
\text { de vegetação (\%) }\end{array}$ \\
\hline 1 & $82 \mathrm{~b}$ & $46 \mathrm{ab}$ & $79 \mathrm{ab}$ \\
2 & $80 \mathrm{~b}$ & $35 \mathrm{~b}$ & $66 \mathrm{~b}$ \\
3 & $88 \mathrm{a}$ & $50 \mathrm{a}$ & $82 \mathrm{a}$ \\
4 & $81 \mathrm{~b}$ & $48 \mathrm{ab}$ & $78 \mathrm{ab}$ \\
\hline $\mathrm{CV}(\%)$ & 4,3 & 5,5 & 6,8 \\
\hline
\end{tabular}

Médias seguidas pela mesma letra na coluna não diferem entre si, teste de Tukey, pd"0,05 (means followed by the same letter in the column did not differ from each other, Tukey's test, $\mathrm{p} \leq 0.05$ ).

água por soluções saturadas de sais durante a realização do teste; reduzindo, dessa forma, a umidade relativa do ambiente no interior dos compartimentos individuais e, conseqüentemente, retardando e uniformizando a absorção de água pelas sementes. Como as sementes de urucum são relativamente pequenas, considera-se que este procedimento possa ser adequado da mesma forma que se mostrou para as de milhodoce (Bennett et al., 1998), melão (Torres \& Marcos Filho, 2003), pepino (Abdo et al., 2005) e trigo (Lima et al., 2006). Por outro lado, este procedimento não se mostrou adequado para a avaliação do vigor de sementes de alface, brócolos e cenoura (Ribeiro, 2001) e de melancia (Bhéring et al., 2003).

O presente trabalho teve como objetivo adequar a metodologia do teste de envelhecimento acelerado para a avaliação do potencial fisiológico de sementes de urucum.

\section{MATERIAL E MÉTODOS}

O experimento foi conduzido em laboratório da Empresa de Pesquisa Agropecuária do Rio Grande do Norte e da Universidade Federal Rural do SemiÁrido em Mossoró (RN), utilizando-se quatro lotes de sementes de urucum, cultivar Casca Vermelha, provenientes de agricultores do estado da Paraíba.

Antes da instalação dos testes, pelo fato de ter sido detectada dormência tegumentar nas sementes, com germinação em torno de $15 \%$ foi realizada superação da dormência, por meio de desponte na região oposta ao eixo embrionário, sendo imediatamente submetidas aos testes: grau de umidade (pelo méto- do estufa a $105 \pm 3^{\circ} \mathrm{C}$ por 24 horas, utilizando-se duas subamostras de sementes, sem desponte, para cada lote, com os resultados expressos em \% (Brasil, 1992)); germinação (realizado com quatro subamostras de 50 sementes, distribuídas sobre papel toalha, tipo "germitest", umedecido com quantidade de água igual a 2,2 vezes seu peso seco e colocadas para germinar a $30^{\circ} \mathrm{C}$ ). As contagens foram realizadas aos sete e dez dias após a semeadura, conforme Custódio et al. (2002); primeira contagem de germinação - constituiu-se dos resultados obtidos na primeira contagem de plântulas normais, realizada no sétimo dia do teste de germinação; emergência de plântulas em casa de vegetação - conduzido com quatro subamostras de 50 sementes, distribuídas em bandejas multicelulares contendo substrato organo-mineral Plantimax $®$ HA, suficientemente umedecido.

As irrigações foram realizadas sempre que necessário. Os resultados foram expressos em \% de plântulas normais obtidas aos 21 dias após a semeadura; envelhecimento acelerado (método tradicional) - adotou-se a metodologia recomendada pela AOSA (1983) e complementada por Marcos Filho (1999). Foram utilizadas caixas de plástico para germinação $(11 \times 11 \times 6 \mathrm{~cm})$, possuindo em seu interior uma bandeja com tela de alumínio onde as sementes, após pesagem $(5,0 \mathrm{~g})$, foram distribuídas de maneira a formar uma camada uniforme. Dentro de cada compartimento individual foram adicionados $40 \mathrm{~mL}$ de água destilada. As caixas tampadas permaneceram no interior da câmara, a $41^{\circ} \mathrm{C}$, por períodos de 48,72 e 96 horas.

Em seguida, as sementes foram colocadas para germinar conforme metodologia descrita para o teste de germinação. A avaliação foi realizada aos sete dias após a semeadura e os resultados expressos em \% média de plântulas normais para cada lote; envelhecimento acelerado com o uso de solução salina saturada - utilizou-se a metodologia descrita para o método tradicional, porém, substituindo-se a água no interior das caixas de plástico para germinação por $40 \mathrm{~mL}$ de solução saturada de $\mathrm{NaCl}$ (40 g de $\mathrm{NaCl}$ em $100 \mathrm{~mL}$ de água), obtendo-se, assim, ambiente com $76 \%$ de UR, conforme descrito por Jianhua \& McDonald (1996).

Após o envelhecimento acelerado (tradicional e modificado), o grau de umidade das sementes foi determinado pelo método da estufa a $105 \pm 3^{\circ} \mathrm{C}$ por 24 horas, sendo os resultados expressos em porcentagem.

O delineamento experimental empregado foi o inteiramente casualizado com quatro repetições. Os dados em porcentagem foram previamente transformados em arc sen $\sqrt{x / 100}$, porém os valores originais foram empregados nas Tabelas. A comparação das médias foi feita pelo teste de Tukey, em 5\% de probabilidade. Os dados referentes ao grau de umidade das sementes não foram submetidos à análise estatística.

\section{RESULTADOS E DISCUSSÃO}

Pelo teste de germinação, verifica-se que apenas o lote 3 foi significativamente superior aos demais (Tabela 1). Já o teste de primeira contagem de germinação mostrou-se mais sensível que o de germinação, classificando os lotes em diferentes níveis de qualidade fisiológica (Tabela 1). Neste teste, observa-se que o lote 3 foi classificado como superior, porém não diferindo estatisticamente dos lotes 1 e 4 . O lote 2 foi considerado como de qualidade inferior. Embora a primeira contagem de germinação possa ser considerada um indicativo de vigor, sabe-se que a redução da velocidade de germinação não está entre os primeiros eventos do processo de deterioração de sementes (Delouche \& Baskin, 1973), justificando a menor eficiência deste teste em detectar pequenas diferenças de vigor e o conseqüente agrupamento dos lotes. 
Por outro lado, para sementes de pepino, Bhéring et al. (2000) verificaram que o teste de primeira contagem de germinação pode ser utilizado rotineiramente para se obter informações preliminares sobre o vigor de lotes de sementes de urucum. O teste de emergência em casa de vegetação possibilitou a classificação dos lotes de forma semelhante ao da primeira contagem de germinação. Na concepção de Marcos Filho (1999) o teste de emergência de plântulas constitui parâmetro indicador da eficiência dos testes para a avaliação do potencial fisiológico de lotes de sementes.

Os quatro lotes de sementes avaliados revelaram graus de umidade inicial semelhantes, variando de 6,5 a 6,9\%. Esse fato é importante para execução dos testes, considerando-se que a uniformização do grau de umidade das sementes é imprescindível para a padronização das avaliações e obtenção de resultados consistentes (Marcos Filho, 1999). O grau de umidade das sementes após o envelhecimento tradicional variou de 23,6 a $38,7 \%$, enquanto para as sementes submetidas ao envelhecimento com solução saturada foi de 10,0 a $10,9 \%$.

Os resultados demonstraram que o uso de solução saturada de $\mathrm{NaCl}$ proporcionou maior uniformidade e retardamento na absorção de água pelas sementes de urucum, conforme proposto por Jianhua \& McDonald (1996). Desta maneira, as condições de envelhecimento com o uso de solução de sal promoveram efeitos menos drásticos, pois ao atingir menores teores de água, o grau de deterioração das sementes foi atenuado em relação ao normalmente verificado com o uso do procedimento tradicional. Outra vantagem adicional do emprego da solução saturada de sal é a redução do desenvolvimento de fungos durante o teste, em função da restrição hídrica da umidade relativa do ambiente no interior das caixas plásticas que não favorece a proliferação de microorganismos. Isto foi confirmado, pois com adição de solução saturada de $\mathrm{NaCl}$ não foi verificada a presença de fungos. Observações semelhantes foram constatadas por Jianhua \& McDonald (1996), em sementes de Impatiens walleriana (maria-sem-vergonha); Ra-

Tabela 2. Porcentagem de plântulas normais obtida no teste de envelhecimento acelerado tradicional (água) e com solução saturada de $\mathrm{NaCl}$, a $41^{\circ} \mathrm{C}$, em sementes de urucum, cultivar Casca Vermelha (normal seedlings percentage obtained after the accelerated aging test with water and $\mathrm{NaCl}$ saturated solution, at $41^{\circ} \mathrm{C}$, in annatto seeds, cultivar Casca Vermelha). Mossoró, UFERSA, 2007.

\begin{tabular}{|c|c|c|c|c|c|c|c|c|c|c|}
\hline \multirow{2}{*}{ Lote } & \multicolumn{6}{|c|}{ Tradicional (água) } & \multicolumn{4}{|c|}{ Solução saturada (NaCl) } \\
\hline & \multicolumn{2}{|c|}{$48 \mathrm{~h}$} & \multicolumn{2}{|r|}{$72 \mathrm{~h}$} & \multicolumn{2}{|c|}{$96 \mathrm{~h}$} & \multicolumn{2}{|c|}{$48 \mathrm{~h}$} & \multirow{2}{*}{$\begin{array}{r}72 h \\
65 b\end{array}$} & \multirow{2}{*}{$\frac{96 h}{63 b}$} \\
\hline 1 & 63 & $a b$ & 54 & $a b$ & 38 & $a b$ & 70 & $a b$ & & \\
\hline 2 & 59 & $\mathrm{~b}$ & 44 & $b$ & 32 & $b$ & 57 & $b$ & $51 \mathrm{c}$ & $59 \mathrm{~b}$ \\
\hline 3 & 72 & a & 58 & a & 40 & a & 72 & a & $78 \mathrm{a}$ & $73 a$ \\
\hline 4 & 65 & $a b$ & 56 & $a b$ & 36 & $a b$ & 67 & $a b$ & $64 \mathrm{~b}$ & $62 b$ \\
\hline CV (\%) & & 6,1 & & 7,2 & & 5,5 & & 5,4 & 6,2 & 4,0 \\
\hline
\end{tabular}

Médias seguidas pela mesma letra na coluna não diferem entre si, teste de Tukey, pd"0,05 (means followed by the same letter in the column did not differ from each other, Tukey's test, $\mathrm{p} \leq 0.05$ ).

mos et al. (2004), em sementes de rúcula; Ávila et al. (2006), em sementes de rabanete e Nascimento et al. (2007), em sementes de ervilha.

À semelhança do que ocorreu nos testes de germinação, primeira contagem e emergência das plântulas em casa de vegetação, as sementes de urucum do lote 3 submetidas ao teste de envelhecimento tradicional e modificado, para ambos períodos de envelhecimento, confirmou o lote 3 como o de melhor qualidade fisiológica (Tabela 2). Os períodos de exposição de 48, 72 e 96 horas, no teste tradicional, obedeceram à mesma tendência dos resultados obtidos no teste de germinação, primeira contagem e emergência. Neste procedimento, verifica-se que o período de exposição de 96 horas foi mais drástico às sementes. Esse efeito provavelmente deve-se ao alto teor de água atingido pelas sementes após o envelhecimento com o uso dessa temperatura. Resultados semelhantes foram verificados por Ramos et al. (2004), em sementes de rúcula e, também, por Costa et al. (2008), em sementes de couve, couve-brócolis e repolho.

Para os resultados referentes ao teste de envelhecimento acelerado com utilização de solução saturada de $\mathrm{NaCl}$ (Tabela 2), verifica-se que o período de 72 horas de envelhecimento provocou a separação dos lotes em diferentes níveis de vigor, confirmando o lote 3 como de melhor qualidade, os lotes 1 e 4 como intermediários e o lote 2 como o de pior qualidade. De forma geral, esses resultados são concordantes com os obtidos nos testes de germinação, primeira contagem e emergência de plântulas. Resul- tados semelhantes foram verificados por Torres (2004), em sementes de erva-doce, e Ávila et al. (2006), em sementes de rabanete, quando utilizaram este mesmo período a $41^{\circ} \mathrm{C}$.

Isso posto, o teste de envelhecimento acelerado com o uso de solução saturada de $\mathrm{NaCl}$ revelou-se eficiente para avaliar o vigor de sementes de urucum, e o período de 72 horas a $41^{\circ} \mathrm{C}$, demonstrou ser o procedimento mais adequado para a classificação dos lotes em diferentes níveis de vigor.

\section{REFERÊNCIAS}

ABDO MTVN; PIMENTA RS; PANOBIANCO M; VIEIRA RD. 2005. Testes de vigor para avaliação de sementes de pepino. Revista Brasileira de Sementes 27: 195-198.

ASSOCIATION OF OFFICIAL SEED ANALYSTS. 1983. Seed vigor testing handbook. East lansing: AOSA. 88p. (Contribution 32).

ÁVILA PFV; VILLELA FA; ÁVILA MSV. 2006. Teste de envelhecimento acelerado para avaliação do potencial fisiológico de sementes de rabanete. Revista Brasileira de Sementes 28: 52-58.

BENNETT M; BARR A; GRASSBAUGH EM; EVANS AF. 1998. Seed vigor evaluation of $s u$, se and $s h 2$ sweet corn genotypes using the saturated salt accelerated aging test. In: INTERNATIONAL SEED TESTING CONGRESS, 25. 1998, Pretoria. Abstracts... Pretoria: ISTA, p.92-93.

BHÉRING MC; DIAS DCFS; BARROS DI; TOKUHISA D. 2003. Avaliação do vigor de sementes de melancia (Citrullus lanatus Scherad) pelo teste de envelhecimento acelerado. Revista Brasileira de Sementes 25: 1-6.

BHÉRING MC; DIAS DCFS; GOMES JM; BARROS DI. 2000. Métodos para avaliação do vigor de sementes de pepino. Revista Brasileira de Sementes 22: 171-175. 
BRASIL. 1992. Ministério da Agricultura e Reforma Agrária. Regras para análise de sementes. Brasília: SNDA/DNDV/CLAV. $365 \mathrm{p}$.

COSTA CJ; TRZECIAK MB; VILLELA FA. 2008. Potencial fisiológico de sementes de brássicas com ênfase no teste de envelhecimento acelerado. Horticultura Brasileira 26: 144-148.

CUSTÓDIO CC; MACHADO NETO NB; CASEIRO RF; IKEDA M; BONFIM DC. 2002. Germinação de sementes de Urucum (Bixa orellana L.). Revista Brasileira de Sementes 24: 197-202.

DELOUCHE JC; BASKIN CC. 1973. Accelerated aging techniques for predicting the relative storability of seed lots. Seed Science and Technology 1: 427-452.

HARDER MNC. 2005. Efeito do urucum (Bixa orellana L.) na alteração de características de ovos de galinhas poedeiras. Piracicaba: USP-ESALQ. 74p. (Tese mestrado).

JIANHUA Z; McDONALD MD. 1996. The saturated salt accelerated aging test for small-seeded crops. Seed Science and Technology 25: 123-131.
LIMA TC; MEDINA PF; FANAN S. 2006. Avaliação do vigor de sementes de trigo pelo teste de envelhecimento acelerado. Revista Brasileira de Sementes 28: 106113.

LOPES JC; LIMA RV; MACEDO CMP. 2008. Germinação e vigor de sementes de urucu. Horticultura Brasileira 26: 19-25.

LORENZI H. 2002. Plantas medicinais no Brasil: nativas e exóticas. Nova Odessa: Instituto Plantarum. p.95-96.

MARCOS FILHO J. 1999. Testes de vigor: importância e utilização. In: KRZYZANOWSKI FC; VIEIRA RD; FRANÇA NETO J.B. (eds.). Vigor de sementes: conceitos e testes. Londrina: ABRATES. cap.1, p.1-21.

NASCIMENTO WM; FREITAS RA; GOMES EMLG; SOARES AS. 2007. Metodologia para o teste de envelhecimento acelerado em sementes de ervilha. Horticultura Brasileira 25: 205-209.

PANOBIANCO M; MARCOS FILHO J. 2001. Envelhecimento acelerado e deterioração controlada em sementes de tomate. Scientia Agricola 58: 525-531.
POWELL AA. 1995. The controlled deterioration test. In: VAN DE VENTER HA. (ed.). Seed vigour testing seminar. Copenhagen: ISTA. p.73-87.

RAMOS NP; FLOR EPO; MENDONÇA EAF; MINAMI K. 2004. Envelhecimento acelerado em sementes de rúcula (Eruca sativa L.). Revista Brasileira de Sementes 26: 98-103.

RIBEIRO FC. 2001. Comparação entre os sistemas de envelhecimento artificial de sementes através de soluções salinas e o tradicional em cenoura (Daucus carota L.) alface (Lactuca sativa L.) e brócolos (Brassica oleracea var. etálica Plenk.). Jaboticabal: UNESP-FCAV. 56p. (Tese mestrado).

TEKRONY DM. 1995. Accelerated aging. In: VAN DE VENTER HA. (ed.). Seed vigour testing seminar. Copenhagen: ISTA. p.5372.

TORRES SB. 2004. Teste de envelhecimento acelerado em sementes de erva-doce. Revista Brasileira de Sementes 26: 20-24.

TORRES SB; MARCOS FILHO J. 2003. Accelerated aging of melon seeds. Scientia Agricola 60: 77-82. 